\title{
A Low-Cost GNSS Prototype for Tracking Runners in RTK Mode: Comparison with Running Watch Performance
}

\author{
Yuri Taddia ${ }^{1}\left(\mathbb{D}\right.$, Luca $_{\text {Ercolin }}^{2}$, and Alberto Pellegrinelli ${ }^{1(\bowtie)}(\mathbb{D}$ \\ ${ }^{1}$ Engineering Department, University of Ferrara, Via Saragat 1, 44122 Ferrara, Italy \\ \{yuri.taddia, alberto.pellegrinelli\} @unife.it \\ 2 Department of Neuroscience and Rehabilitation, Faculty of Medicine, \\ Pharmacy and Prevention, University of Ferrara, Via Borsari 46, 44121 Ferrara, Italy \\ luca.ercolin@unife.it
}

\begin{abstract}
GNSS positioning is widely use in every kind of application. Nowadays, low-cost GNSS modules are becoming available to apply the Real-Time Kinematic mode in those applications in which a centimeter-level accuracy would be appreciated for a precise positioning. In this work, we developed a prototype for collecting data in RTK mode with a single-frequency multi-constellation device during some physical tests performed by a professional runner. Prior to do this, we assessed the accuracy in estimating the distance actually covered during a walking on a signalized line. Also, we verified the capability to detect short sprints of about 12-15 s. Finally, we compared the results of our prototype with a Polar M430 running watch during three Cooper tests and a Kosmin test. The comparison highlighted that the running watch overestimated the total distance systematically and did not describe the performance of the athlete accurately in time. The distance overestimation was $+4.7 \%$ on average using the running watch, whereas our prototype system exhibited an error level of about $0.1 \%$.
\end{abstract}

Keywords: Low-cost GNSS RTK · Running watch · Cooper test · Kosmin test · u-blox

\section{Introduction}

Global Positioning System (GPS) has been widely implemented during last decades in almost every field of our life. Car navigation based on the use of standard GPS modules is one of the most common applications since decades. Nowadays, Global Navigation Satellite System (GNSS) positioning, with more constellations than GPS only (GLONASS, Galileo, Beidou) is available on a wide range of devices, such as smartphones for instance. However, the miniaturization of antennas and receivers has spread the use of GNSS positioning also to physical activity and sports. The standard accuracy level of some meters is certainly suitable for car navigation since, essentially, the real position is bound to be on the road. Also, the path followed during hikes or similar non-human activities, such as tracking dogs in the streets [1], is still well described using

(C) The Author(s) 2022

E. Borgogno-Mondino and P. Zamperlin (Eds.): ASITA 2021, CCIS 1507, pp. 233-245, 2022.

https://doi.org/10.1007/978-3-030-94426-1_17 
standard GNSS positioning. However, a higher level of accuracy is needed whenever the GNSS data collection is further used to evaluate the actual performance of athletes for scheduling training activities.

Nowadays, low-cost GNSS devices are available for mass market. Such instruments are based on small modules able to receive not only C/A codes, but also to perform carrier phase measurements. When this kind of approach is combined to the use of a second module placed on a fixed position, it is possible to operate in Real-Time Kinematic (RTK) mode obtaining a final positioning characterized by a centimeter-level accuracy in optimal conditions [2]. U-blox modules implement this technology and make it available through application boards ready to be integrated in prototypes for almost every kind of positioning-based application [3, 4]. High-precision single-frequency in RTK mode has been successfully developed and validated for vehicle applications [5] and non-human tracking [6]. The development of a small and lightweight system for applying the GNSS RTK positioning to running, both from the point of view of the professionalism and the amateurism, can help to describe in a better manner the performance level of athletes. In fact, test analysis often starts from the total distance covered by the runner to establish which is the actual degree of preparation of the athlete for a certain kind of competition. This is the case of Cooper and Kosmin tests. Cooper test [7], is a way to analyze the performance of an athlete who has a total time of $12 \mathrm{~min}$ available and has to cover the longest distance as possible managing its own physical resources. Kosmin test, instead, is predictive for middle-distance competitions and consists of a sequence of running (as fast as possible) and stops (recovery time). The sequence for predicting $1500 \mathrm{~m}$ test is: 1-min running, 3-min stop, 1-min running, 2-min stop, 1-min running, 1-min stop and final 1-min running. For instance, Cooper test followed by GPS tracking during football matches has been used to analyze physical performance in adolescents [8] and GPS with accelerometry data to assess benefits of physical activity in seniors [9].

Evaluating the distance with a high level of accuracy can hence make the difference: a wrong classification of the performance with respect to the real one due to too large measurement uncertainties can lead, in the worst scenario, up to muscle strains because of an overexertion. In this research, we therefore developed a simple prototype with the aim of survey the performance of a professional runner during the execution of some technical tests (Cooper and Kosmin). Also, we investigated the level of accuracy obtainable using the prototype, as well as we compared it with some running watches commonly used by the athletes to evaluate their own performance.

\section{Materials and Methods}

In this work, we used the u-blox RTK application board package C94-M8P, based on u-blox NEO-M8P-2 module, to develop an easily wearable GNSS rover prototype for tracking the path of an athlete. In particular, our system consists of one C94-M8P application board with NEO-M8P-2 module as GNSS receiver, an external GNSS antenna and an external UHF antenna. Both antennas are connected to the main board. The GNSS antenna is fixed to a bike helmet that is worn by the runner during each measurement session. The application board is also connected to a Raspberry Pi 3 Model B+ where the data flow of GNSS solutions epoch per epoch is continuously recorded. The Raspberry 
$\mathrm{Pi}$ is powered by a compact battery bank and the overall system is contained into a small backpack easily wearable by runners (see Fig. 1).

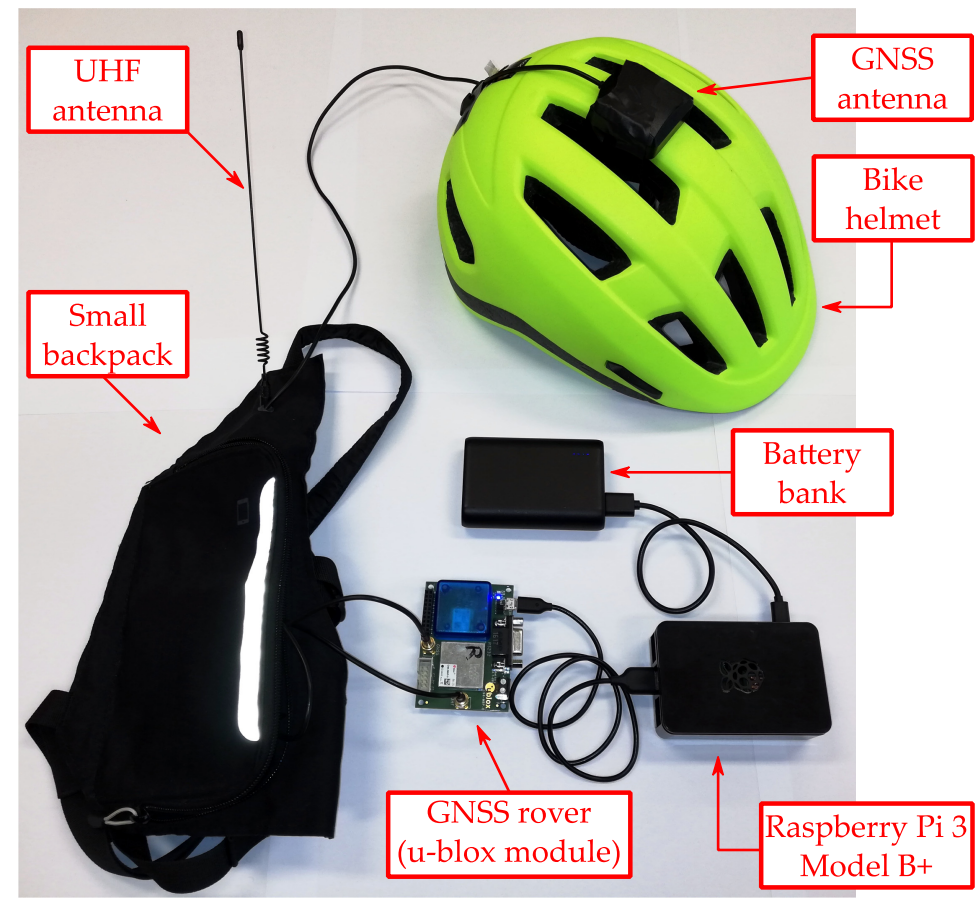

Fig. 1. Description of the wearable GNSS rover prototype system.

At the same time, another application board, acting as a base station for RTK operations, is set up every time. The location of the base station is arbitrary and a survey-in procedure starts every time the base receiver is powered on. After $3 \mathrm{~min}$, the position is assumed as fixed and hence the base starts sending data to the rover through UHF radio for reaching a rover solution in which the carrier phase ambiguities have been fixed. Both the GNSS receivers are single-frequency, multi-constellation and were set up to operate as GPS + GLONASS in RTK mode. The maximum RTK solution rate we tested is $5 \mathrm{~Hz}$, with a solution every $0.2 \mathrm{~s}$. However, in the following we use a rate of $1 \mathrm{~Hz}$ with the aim to perform a direct comparison with the data collected using running watches. All the tests were carried out in a running track, placing the base receiver in the lawn inside the track. Hence, the maximum base-rover distance (i.e., the baseline) never exceeded $100 \mathrm{~m}$. No significant obstacles reduced the sky visual, with the only exception of some buildings with a height of $15 \mathrm{~m}$ located at about $20 \mathrm{~m}$ from one side of the track, that is a common situation for urban running tracks.

Prior to use our prototype on a real athlete, we carried out two preliminary tests. The first consisted in analyzing the lateral uncertainty level when walking along a signalized path around the running track. The prototype (also simply referred to as u-blox in the following) was hence worn as reported in Fig. 2 and the test was conducted with a low 
speed, following a specific path. The same line, signalized on the ground, was surveyed using a geodetic GNSS receiver (Topcon GR-3) in NRTK mode with the dual purpose to reconstruct the horizontal path and computing its real length. We therefore computed the lateral deviations for each RTK solution (Fig. 4).

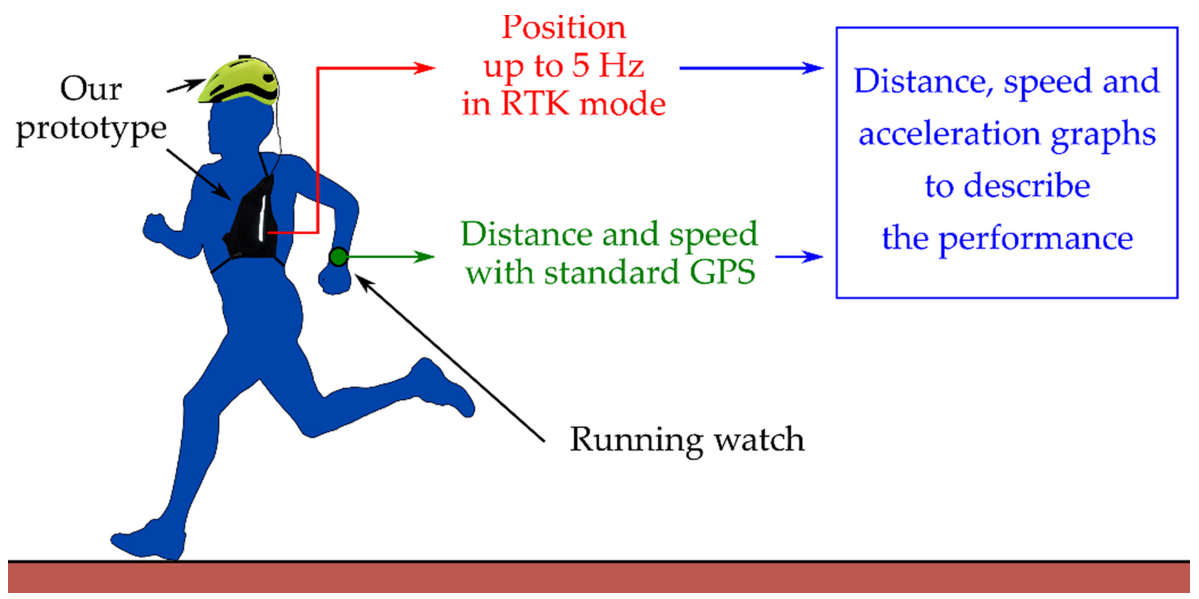

Fig. 2. Data acquisition with both systems worn by the athlete.

The second test aimed to compare the performance of our prototype with those of a common running watch (TomTom Runner 2 Cardio, abbreviated as TomTom in the following), simulating short sprints and analyzing the capability of both devices in detecting them. The position of the runner was hence collected simultaneously. Four sprints were simulated during this test. Sprint durations were ca. $15 \mathrm{~s}$ for the first two sprints and ca. $12 \mathrm{~s}$ for the last ones. The scheme is also reported in Fig. 3. Distance, speed and acceleration have been computed afterwards. The aim was to evaluate whether the use of our prototype introduced any improvement in describing accelerations and decelerations in short time-frames.

Finally, we collected data during Cooper tests performed by a professional athlete. It is worth noting here that our prototype has been developed with a particular regard to not interact with the performance of the runner. The procedure we applied for acquiring data essentially consisted in powering on both boards every time: in fact, they were set up to operate as a base and a rover respectively. Data recording on the Raspberry Pi automatically starts at power on. The fix of carrier phase ambiguities on the rover is ensured by a led indicator.

A total amount of three Cooper tests and one Kosmin test were surveyed and analyzed in order to assess the difference in using a running watch and our prototype. A high-end Polar M430 device was used instead of the TomTom previously employed during the preliminary comparison. In this work, we aimed to reconstruct the path followed by the runner with a time step of $1 \mathrm{~s}$. Polar M430 directly gave distance and speed as output, 
whereas our prototype was set to send NMEA GGA strings as output data flow. From such strings, we finally computed East and North coordinates (UTM projection). Linear deformation module was also taken into account when computing the real distance from horizontal UTM coordinates.

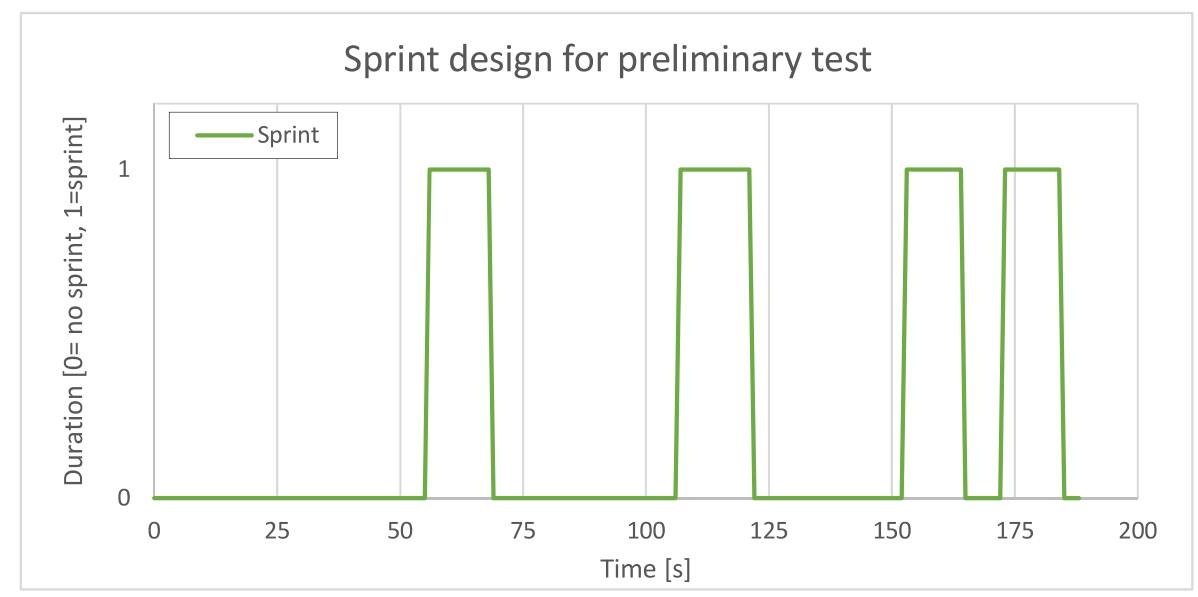

Fig. 3. Duration of sprints during the second preliminary test.

\section{Results}

The first preliminary test, conducted on a track line, highlighted that the $68 \%$ of the lateral deviations were in the range of $0-0.107 \mathrm{~m}$ and the $95 \%$ in the range of $0-0.25 \mathrm{~m}$ (Fig. 4). The total number of points that was collected during the test is 478 . The total distance covered was twice a lap. The distance computed from the position sampled every second with our prototype showed a mean lap length of $406.06 \mathrm{~m}$. If compared to the real length surveyed using a geodetic receiver $(405.69 \mathrm{~m})$, the error is $+0.1 \%$.
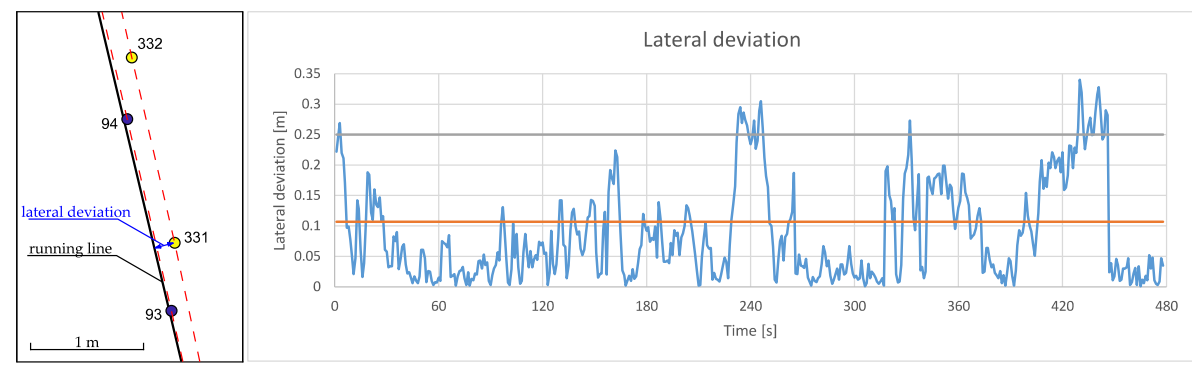

Fig. 4. Lateral deviations from a line during the first preliminary test. 
As mentioned in Sect. 2, we also conducted a second preliminary test focusing on the comparison of a TomTom running watch and our prototype. The results, in terms of distance, speed and acceleration graphs are illustrated in Fig. 5, Fig. 6 and Fig. 7 respectively. It is worth noting, especially looking at Fig. 6, how the wristwatch recognized only two of the four sprints made by the athlete, whereas our prototype recorded higher speeds for each of the actual sprints. This fact is further confirmed analyzing the graph in Fig. 7, which shows the acceleration at the time of each sprint start and the corresponding deceleration at the end of it. Acceleration have been computed from the positions recorded through our prototype and the speeds recorded by the TomTom.

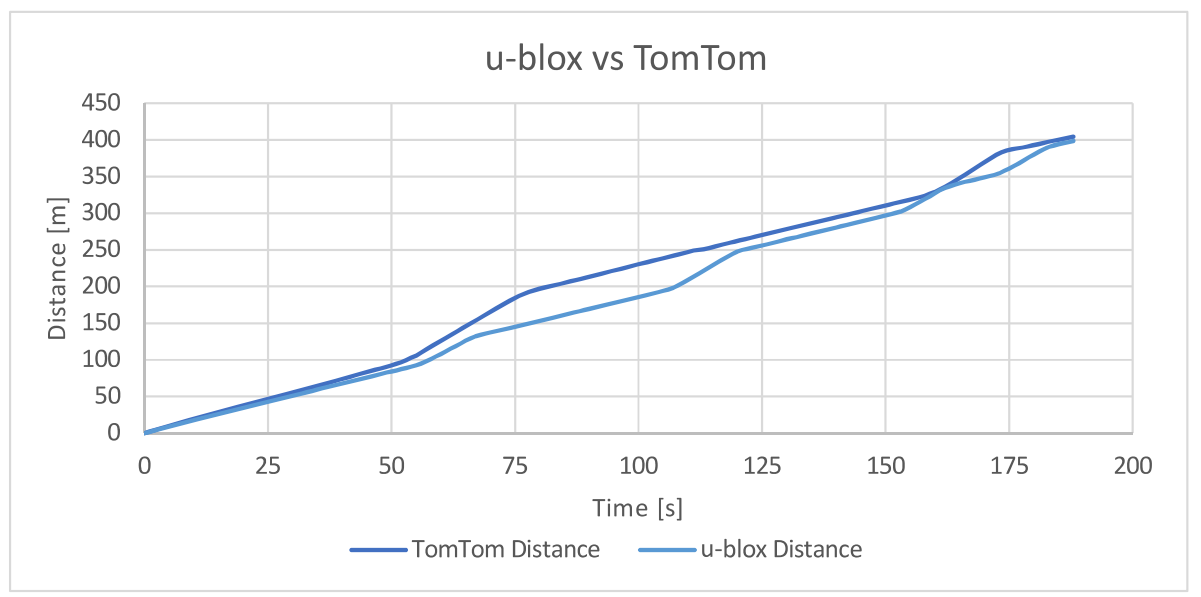

Fig. 5. Distance during preliminary test.

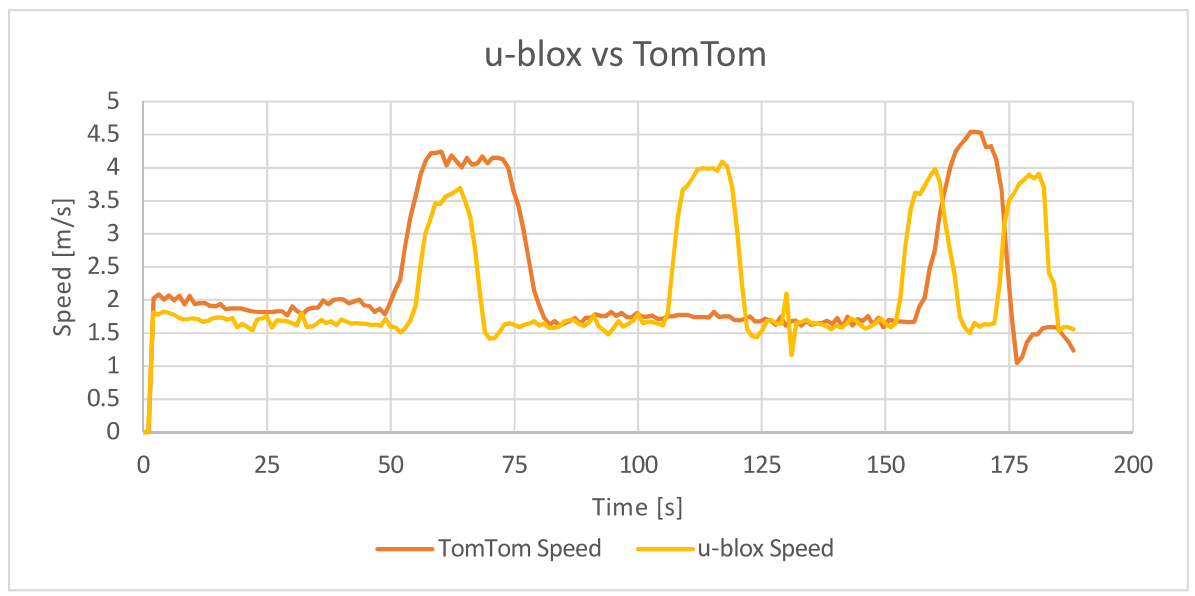

Fig. 6. Speed during preliminary test. 


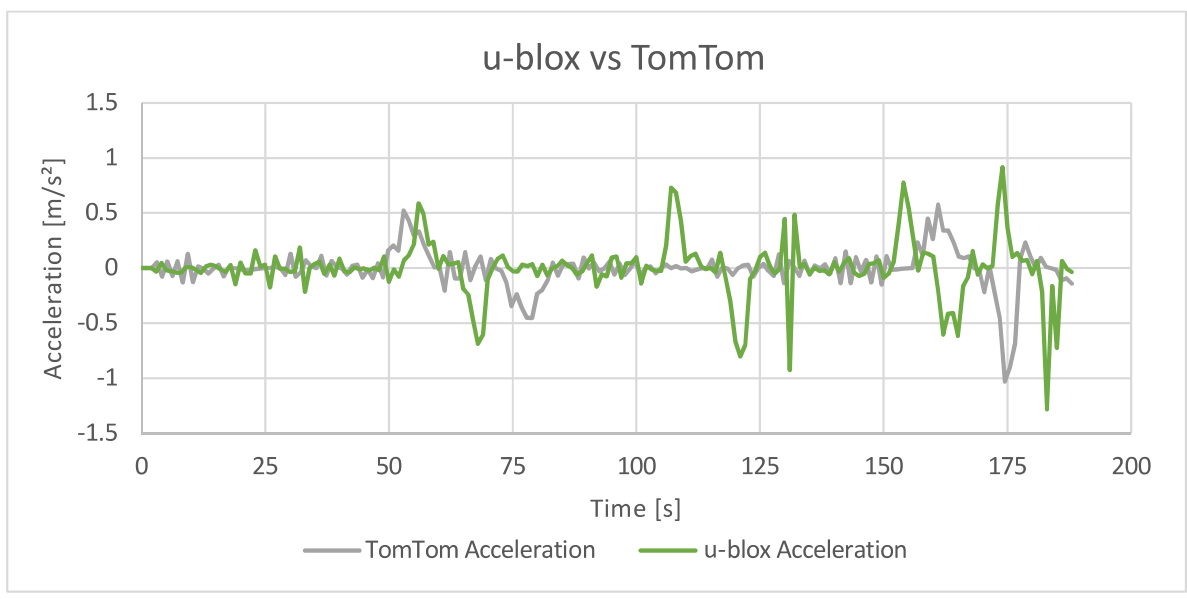

Fig. 7. Acceleration during preliminary test.

Moreover, the duration of the sprints detected by the running watch seems not to be consistent with the actual duration of them. This is clearly noticeable from Fig. 6 and also leads to a deviation of the distance covered from the runner up to ca. $40 \mathrm{~m}$ in about $100 \mathrm{~s}$ from the start of the test (see Fig. 5). However, at the end of the test, the distance difference decreased to less than $1 \mathrm{~m}$.

The data collection made during the performance of Cooper tests is depicted in Fig. 8, Fig. 9 and Fig. 10 in terms of speed versus time and in Fig. 11, Fig. 12 and Fig. 13 in terms of speed versus total distance covered.

Analyzing the graph of speed versus time, it is possible noting that the running watch (Polar M430 in this case) tends to overestimate the real speed of the runner for almost all the duration of the tests. As a consequence, also the distance covered by the athlete was systematically overestimated using the Polar M430.

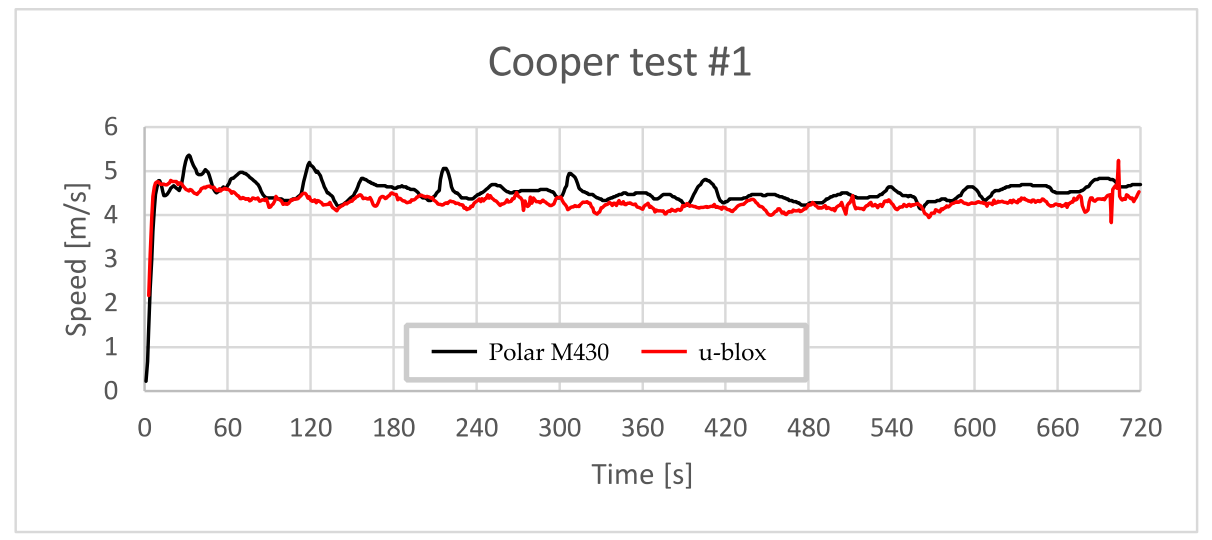

Fig. 8. First Cooper test. 
The same situation is present looking at the graph of Kosmin test (Fig. 14) where the total distance covered during the 4 min of actual running, thus the distance at the end of the test, is still overestimated. In this case, however, it is also very interesting to highlight that the trend of the speed during each running minute is described accurately only with our prototype, especially for the first minute. In fact, at the beginning, the athlete is able to run faster. Hence, there is no reason why the speed should not actually decrease in time as showed from data acquired using our prototype.

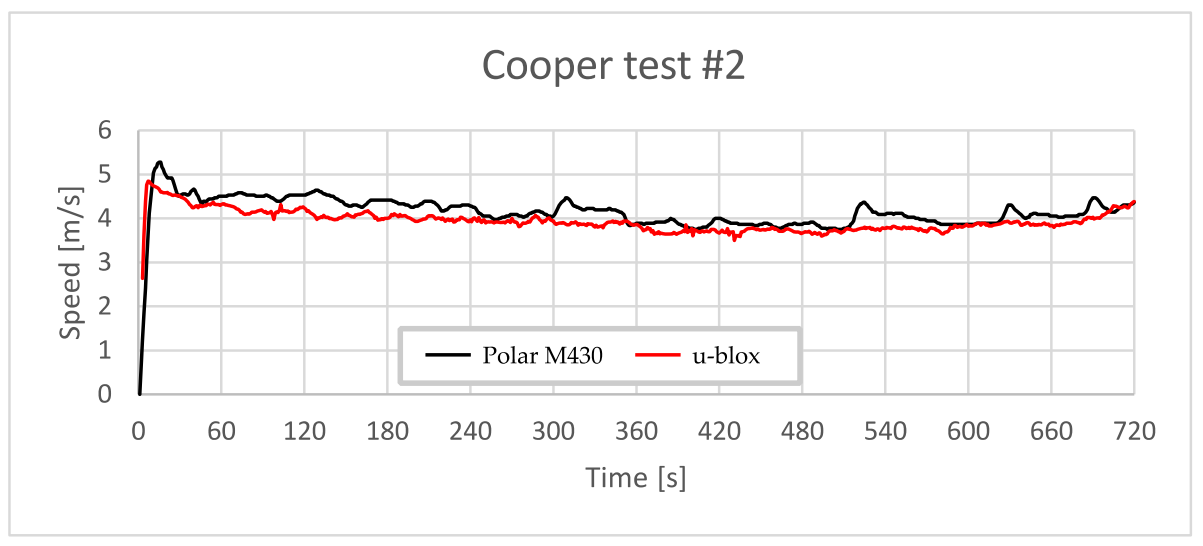

Fig. 9. Second Cooper test.

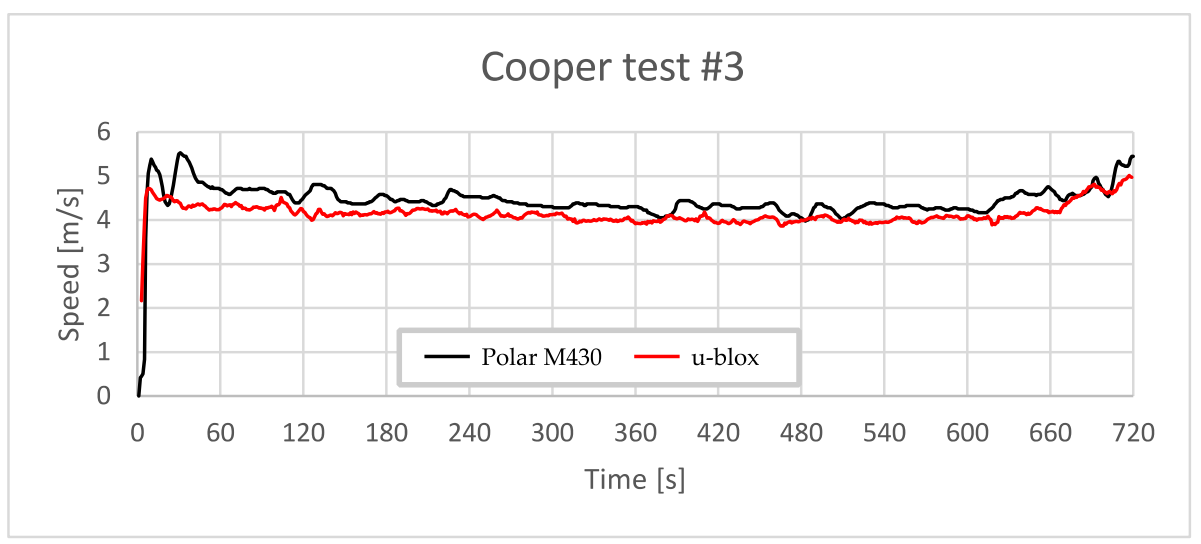

Fig. 10. Third Cooper test. 


\section{Cooper test \#1}

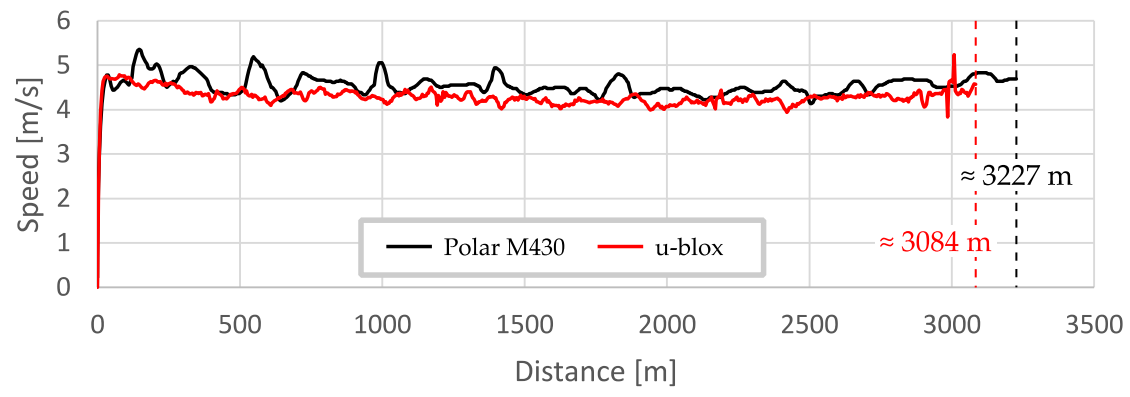

Fig. 11. First Cooper test: total distance.

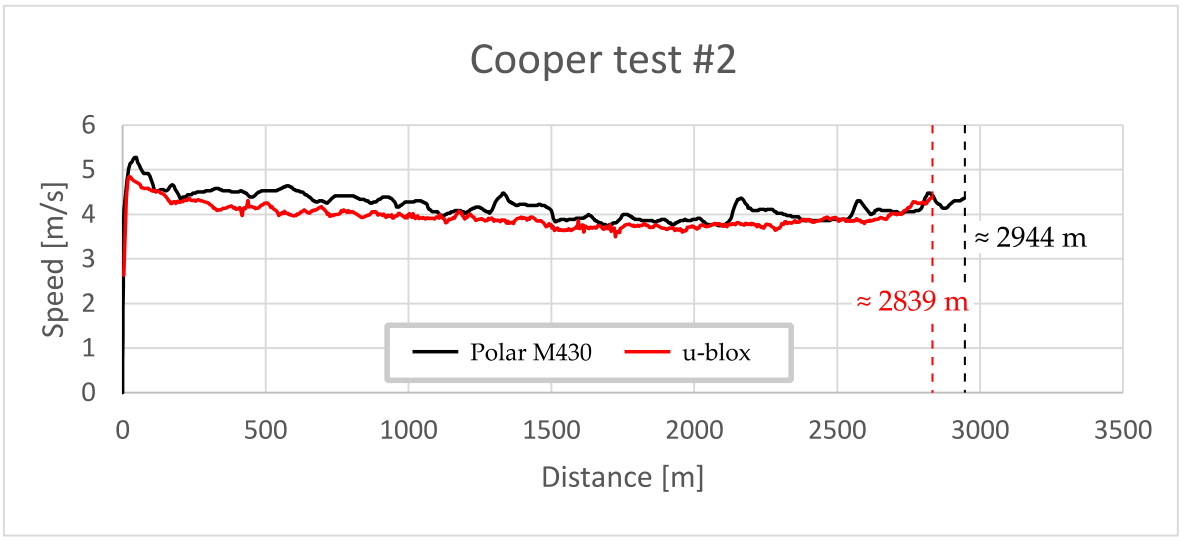

Fig. 12. Second Cooper test: total distance.

\section{Cooper test \#3}

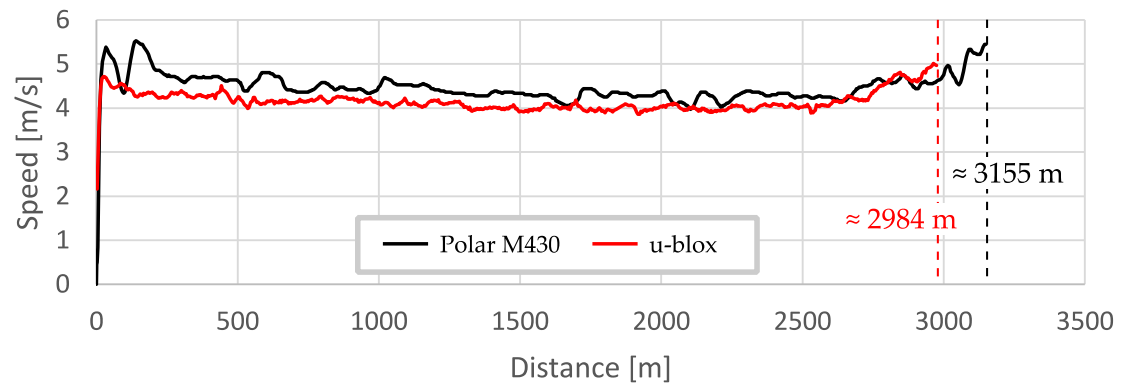

Fig. 13. Third Cooper test: total distance. 


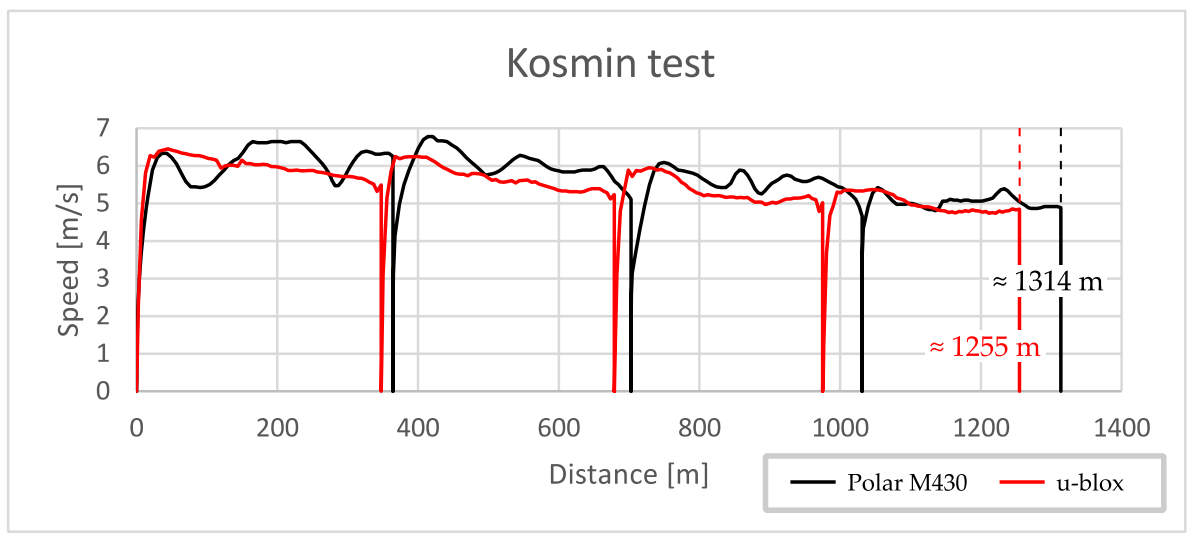

Fig. 14. Kosmin test.

\section{Discussion}

In this work, we presented the first results obtained using a prototype based on low-cost GNSS RTK modules for collecting data about the performance of athletes. In particular, we realized a simple data acquisition system easily wearable in which the antenna is fixed to a bike helmet and both the receiver and a recording unit are contained into a small backpack. First, we conducted a couple of preliminary tests to investigate the level of accuracy of the system and its capability to describe also short sprints (duration of ca. 12-15 s). The first test highlighted that our prototype, compatibly with the ability of a person to follow a signalized line during walking, did not exceed a lateral deviation of more than $0.25 \mathrm{~m}$ for $95 \%$ of the recorded positions. Moreover, the total error in estimating the distance actually covered was of about $+0.1 \%$, thus very accurate. The second test, in which we compared our prototype with a running watch, showed that the sprints were recognized using our prototype, but they were not correctly identified using the running watch. Also, the distance computed using the two different devices highlighted a gap of more than $40 \mathrm{~m}$ in the middle of the test, even if at the end the difference was in the order of a meter. This implies that may be hard to describe the performance of the athlete accurately for all the duration of the test relying on the output of the running watch only.

After these encouraging results obtained in such preliminary tests, we used our prototype on a real runner. We therefore collected data during a series of Cooper tests. In every test, we found an overestimation of the actual speed of the athlete, for all the duration of it, using the Polar M430. This fact causes also an overestimation of the distance covered by the runner, which represents an indicator of the degree of preparation actually reached. This implies that even if the comparison of the performance over time can be still good when affected by an overestimation of the total distance, this is not the case of the classification of the performance in absolute terms. Table 1 reports the distances evaluated from the data recorded by our prototype and the running watch Polar M430. The overestimation using the running watch is $+4.7 \%$ on average $(+140 \mathrm{~m}$ in absolute terms). Such error can make the difference in the evaluation of the real result 
of a Cooper test and about the further considerations regarding the preparation of the runner. It is also worth noting that an actual feedback on the substantial accuracy of the distance estimated using our prototype was given by the fact that the last recorded position was not close to the finish line as pointed out by the data recorded by the Polar M430 (Polar M430 final distance for test \#3 is close to $3200 \mathrm{~m}$, thus almost at the end of the $8^{\text {th }}$ lap theoretically). Finally, we also collected data during a Kosmin test. Once again, we found a difference of $+4.7 \%$ in relative terms between the use of a running watch and our prototype (see Table 1).

Table 1. Comparison of total distance after Cooper tests with our prototype (u-blox) and Polar M430.

\begin{tabular}{l|l|l|l|l}
\hline Test & $\begin{array}{l}\text { u-blox } \\
\text { dist. [m] }\end{array}$ & $\begin{array}{l}\text { Polar M430 } \\
\text { dist. [m] }\end{array}$ & \multicolumn{2}{l}{ Polar M430 overestimation } \\
\hline Cooper \#1 & 3084 & 3227 & $143 \mathrm{~m}$ & $+4.64 \%$ \\
\hline Cooper \#2 & 2839 & 2944 & $105 \mathrm{~m}$ & $+3.70 \%$ \\
\hline Cooper \#3 & 2984 & 3155 & $171 \mathrm{~m}$ & $+5.73 \%$ \\
\hline Kosmin & 1255 & 1314 & $59 \mathrm{~m}$ & $+4.70 \%$ \\
\hline
\end{tabular}

It is worth noting that the Polar M430 is a running watch able to receive only GPS signals and is worn on the wrist, which is not an optimal antenna location. Moreover, positioning algorithms estimating the athlete's path from previous epochs when the signal is partially or temporarily lost are implemented in running watches and excluding them is usually not possible. More recent multi-constellation running watches are available on the market (e.g., GPS + GLONASS), however pseudorange-based standalone positioning has a level of accuracy lower than RTK positioning. The results of this work show that our prototype, based on a GNSS RTK approach, is able to provide a very high level of accuracy in describing the performance of athletes. Positions are recorded every second with a theoretical centimeter-level accuracy. Speed is computed afterwards from the distance between positions at each time.

\section{Conclusions}

This work presented the first results in using a wearable prototype based on GNSS RTK for the data collection aimed to describe the performance of athletes in a more accurate way than using common running watches. Preliminary tests conducted in a running track show an accuracy of about $0.1 \%$ in total distance estimation when using our prototype. Differences in estimating the total distance during both Cooper tests and Kosmin test is instead in the order of $+5 \%$ using a common model of running watch, if compared with our prototype. This highlights the importance of developing systems to acquire more accurate data when using GNSS-based device. The implementation of RTK mode in a compact device can represent an optimal and very promising solution for this. A local base receiver can be established permanently in a training center or, alternatively, a 
Network RTK (NRTK) approach can be adopted. More recent low-cost multi-frequency [10] multi-constellation modules can also be used to increase the potential of the system for reaching fixed solutions. Also, the miniaturization of prototypes is certainly a point to be improved in order to make this technology available for real athletes.

Acknowledgements. The authors are grateful to the students Silvia Sangalli (runner) and Andreas Piva (support during data collection using the prototype) for their contribution during the tests and to Andrea De Vivo (UISP) for having made available to us the running track (test site).

\section{References}

1. Paula, P.M.C., Secco, R.A., Molento, C., Filho, P.L.: Construction of a prototype for tracking dogs in the streets using Arduino. Acta Vet. Brno 14, 61-67 (2020)

2. Garrido-Carretero, M.S., de Lacy-Pérez de los Cobos, M.C., Borque-Arancón, M.J., RuizArmenteros, A.M., Moreno-Guerrero, R., Gil-Cruz, A.J.: Low-cost GNSS receiver in RTK positioning under the standard ISO-17123-8: a feasible option in geomatics. Measur. J. Int. Measur. Confederation 137, 168-178 (2019)

3. Poluzzi, L., Tavasci, L., Corsini, F., Barbarella, M., Gandolfi, S.: Low-cost GNSS sensors for monitoring applications. Appl. Geomat. 12(1), 35-44 (2019). https://doi.org/10.1007/s12 518-019-00268-5

4. Cina, A., Piras, M.: Performance of low-cost GNSS receiver for landslides monitoring: test and results. Geomat. Nat. Haz. Risk 6(5-7), 497-514 (2015)

5. De Bakker, P.F., Tiberius, C.C.J.M.: Single-frequency GNSS positioning for assisted, cooperative and autonomous driving. In: 30th International Technical Meeting of the Satellite Division of the Institute of Navigation, ION GNSS 2017, vol. 6, pp. 4038-4045 (2017)

6. Keshavarzi, H., Lee, C., Johnson, M., Abbott, D., Ni, W., Campbell, D.L.M.: Validation of real-time kinematic (RTK) devices on sheep to detect grazing movement leaders and social networks in merino ewes. Sensors 21(3), 1-19, art. no. 924 (2021)

7. Alvero-Cruz, J.R., Giráldez García, M.A., Carnero, E.A.: Reliability and accuracy of Cooper's test in male long distance runners. Revista Andaluza de Medicina del Deporte 10(2), 60-63 (2017)

8. Gawrecki, A., Michalak, A., Gałczyński, S., Dachowska, I., Zozulińska-Ziółkiewicz, D., Szadkowska, A.: Physical workload and glycemia changes during football matches in adolescents with type 1 diabetes can be comparable. Acta Diabetol. 56(11), 1191-1198 (2019). https://doi.org/10.1007/s00592-019-01371-0

9. Marquet, O., MacIejewska, M., Delclòs-Alió, X., Vich, G., Schipperijn, J., Miralles-Guasch, C.: Physical activity benefits of attending a senior center depend largely on age and gender: a study using GPS and accelerometry data. BMC Geriatr. 20(1), 1-10, art. no. 134 (2020)

10. Hamza, V., Stopar, B., Ambrožič, T., Turk, G., Sterle, O.: Testing multi-frequency low-cost GNSS receivers for geodetic monitoring purposes. Sensors 20(16), 1-16, art. no. 4375 (2020) 
Open Access This chapter is licensed under the terms of the Creative Commons Attribution 4.0 International License (http://creativecommons.org/licenses/by/4.0/), which permits use, sharing, adaptation, distribution and reproduction in any medium or format, as long as you give appropriate credit to the original author(s) and the source, provide a link to the Creative Commons license and indicate if changes were made.

The images or other third party material in this chapter are included in the chapter's Creative Commons license, unless indicated otherwise in a credit line to the material. If material is not included in the chapter's Creative Commons license and your intended use is not permitted by statutory regulation or exceeds the permitted use, you will need to obtain permission directly from the copyright holder.

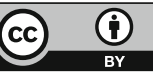

